\title{
Krisenprävention durch Klima- und Energiesicherheit
}

\section{Zeit für Weichenstellungen}

\author{
Christoph Bals, Rixa Schwarz*
}

\begin{abstract}
The climate, food and energy crises have shown the relevance of climate, energy and food security. Tackling these three threats together would serve the prevention of future crises and thus foster human security. Co-operation is another key aspect of such a strategy. The EU has made first steps since 2007 towards handling energy and climate security with an ambitious energy and climate package. So far the current economic crisis has weakened but not fundamentally changed that approach. The next challenge for the new climate and energy leadership role of the EU is the financial package for the Copenhagen agreement at the end of 2009. The March EU Council did not send the expected strong signal and the challenge for the new climate and energy security strategy of the EU has not been yet met. But the ambition of climate action of developing countries also depends on the level of financial and technology co-operation.
\end{abstract}

Keywords: Klimasicherheit, Energiesicherheit, Ernährungssicherheit, Kooperation

Climate security, energy security, nutrition security, cooperation

\section{Klimawandel als Bedrohung menschlicher Sicherheit}

Z iel der UN-Klimarahmenkonvention (Art. 2, UNFCCC - United Nations Framework Convention on Climate Change) ist die Vermeidung eines gefährlichen Klimawandels. Die 1992 verabschiedete Konvention hat es sich also zur Aufgabe gemacht, die Eskalation des anthropogenen Klimawandels soweit zu vermeiden, dass er nicht zum Problem für die menschliche, ${ }^{1}$ nationale und internationale Sicherheit wird. Der Weltklimarat IPCC, die höchste wissenschaftliche Autorität bei dieser Frage, hat 2007 im Vierten Sachstandsbericht ${ }^{2}$ verdeutlicht, dass mit 90- bis 99-prozentiger Wahrscheinlichkeit ${ }^{3}$ vom Menschen freigesetzte Treibhausgase die Hauptursache der globalen Erwärmung der letzten 50 Jahre sind. Extremwetterlagen (Hitzewellen, Starkregen) werden demnach weiter zunehmen und wahrscheinlich werden tropische Stürme (Taifune und Hurrikans) stärker ausfallen. Regenfälle werden in nördlichen Breiten zu-, in den Subtropen dagegen abnehmen. Auch schleichende Veränderungen wie der Anstieg des Meeresspiegels, Gletscherschmelze oder die Ausweitung von Wüsten können die menschliche Sicherheit bedrohen. Der Wissenschaftliche Beirat der Bundesregierung Globale Umweltveränderungen hat vier zentrale klimabedingte Konstellationen identifiziert, die zu Konflikten führen können: „Degradation von Süßwasserressourcen“, „Rückgang der Nahrungsmittelproduktion“, „Zunahme von Sturm- und Flutka-

* Christoph Bals ist Politischer Geschäftsführer bei Germanwatch e.V. in Bonn, Rixa Schwarz ist Referentin für Klima und Sicherheit bei Germanwatch e.V. in Bonn.

1 Das Entwicklungsprogramm der Vereinten Nationen UNDP hat 1994 das Konzept der menschlichen Sicherheit vorgestellt. Die von Amaryata Sen geleitete Commission on Human Security hat diesen Begriff 2003 aufgegriffen und konkretisiert Zwar bleibt der Staat demnach der entscheidende Garant von Sicherheit. Da jedoch die Staaten häufig ihren Verpflichtungen nicht nachkommen oder selbst zum Sicherheitsrisiko werden, "muss sich das Augenmerk von der Sicherheit des Staates zur Sicherheit der Menschen verschieben - zur menschlichen Sicherheit".

2 IPCC - Intergovernmental Panel on Climate Change 2007: Mitigation of Climate Change. Contribution of Working Group III to the Fourth Assessment Report of the IPCC. Genf.

3 Im Jahr 2001 hatte der IPCC die Wahrscheinlichkeit mit 66 bis 90 Prozent eingeschätzt. tastrophen“ und „Umweltbedingte Migration“4. Als regionale Brennpunkte werden Nordafrika, die Sahelzone, das südliche Afrika, Zentralasien, Indien, Pakistan und Bangladesh, China, die Karibik und der Golf von Mexiko sowie die Andenregion und Amazonien angeführt. Diese Aufzählung zeigt: Betroffen sind in erster Linie die ärmeren Länder und dort insbesondere die ärmsten Bevölkerungsgruppen. Der Klimawandel ist bereits heute $^{5}$ ein zusätzlicher Stressfaktor für fragile Staaten ${ }^{6}$.

In jüngster Zeit ist in wissenschaflichen Studien eine Reihe sicherheitsrelevanter Kipp-Elemente identifiziert worden.

Dies sind vom Klimawandel angestoßene Veränderungen, die ganze Kontinente destabilisieren könnten. Zudem wurde geschätzt, wie die Temperaturen von 2000 weiter ansteigen müssten, um diese Veränderungen irreversibel, wenn auch je nach Einzelfall zeitversetzt, auszulösen. Ein irreversibler Schmelzprozess des Eispanzers in Grönland könnte durch einen weiteren Anstieg von ein bis zwei Grad mit der Folge von zwei bis sieben Metern Meeresspiegelanstieg im Laufe von 300 Jahren bewirkt werden. Bei einer Erwärmung um drei bis vier Grad könnten eine allmähliche Intensivierung des El-NiñoEffekts mit Dürren insbesondere in Südostasien, bei drei bis fünf Grad innerhalb von 50 Jahren ein Kollaps des AmazonasRegenwalds, innerhalb von 100 Jahren ein Kollaps des Nordatlantikstroms, der Zentralheizung Mitteleuropas, eintreten. ${ }^{7}$ Die Besorgnis über ein weiteres Kipp-Element, das mögliche rapide Abschmelzen der Gletscher im Himalaya, des „Wasserturms Asiens “, hat Al Gore bei der Senatsanhörung Ende Januar 2009 so zum Ausdruck gebracht: „Die großen Flüsse Asiens, der Indus, der Ganges, der Brahmaputra und der Salween oder der Ayeyarwady, der Mekong, der Jangtse und der Huangho - alle

4 WBGU, 2007: Welt im Wandel: Sicherheitsrisiko Klimawandel, Berlin.

5 Im Juni 2007 bezeichnete UN-Generalsekretär Ban Ki Moon den Darfur-Konflikt als "ersten Klimakrieg". Mag diese Bezeichnung auch überzogen sein, so ist unzweifelhaft das Klima einer der Stressfaktoren im "Netz der Konfliktursachen" (World Commission on Environment and Development: Our Common Future, 1987. Oxford, New Oxford University Press, 9, S. 291), die zur Destabilisierung beigetragen haben.

6 WBGU, 2007: Welt im Wandel: Sicherheitsrisiko Klimawandel, Berlin.

7 Lenton, T. M, Held, H., Kriegler, E., Hall, J.W., Lucht, W., Rahmstorf, S., and Schellnhuber, H.J.:Tipping elements in the Earth's climate system, PNAS, February 12,2008 , vol. 105 , no. 6 . 


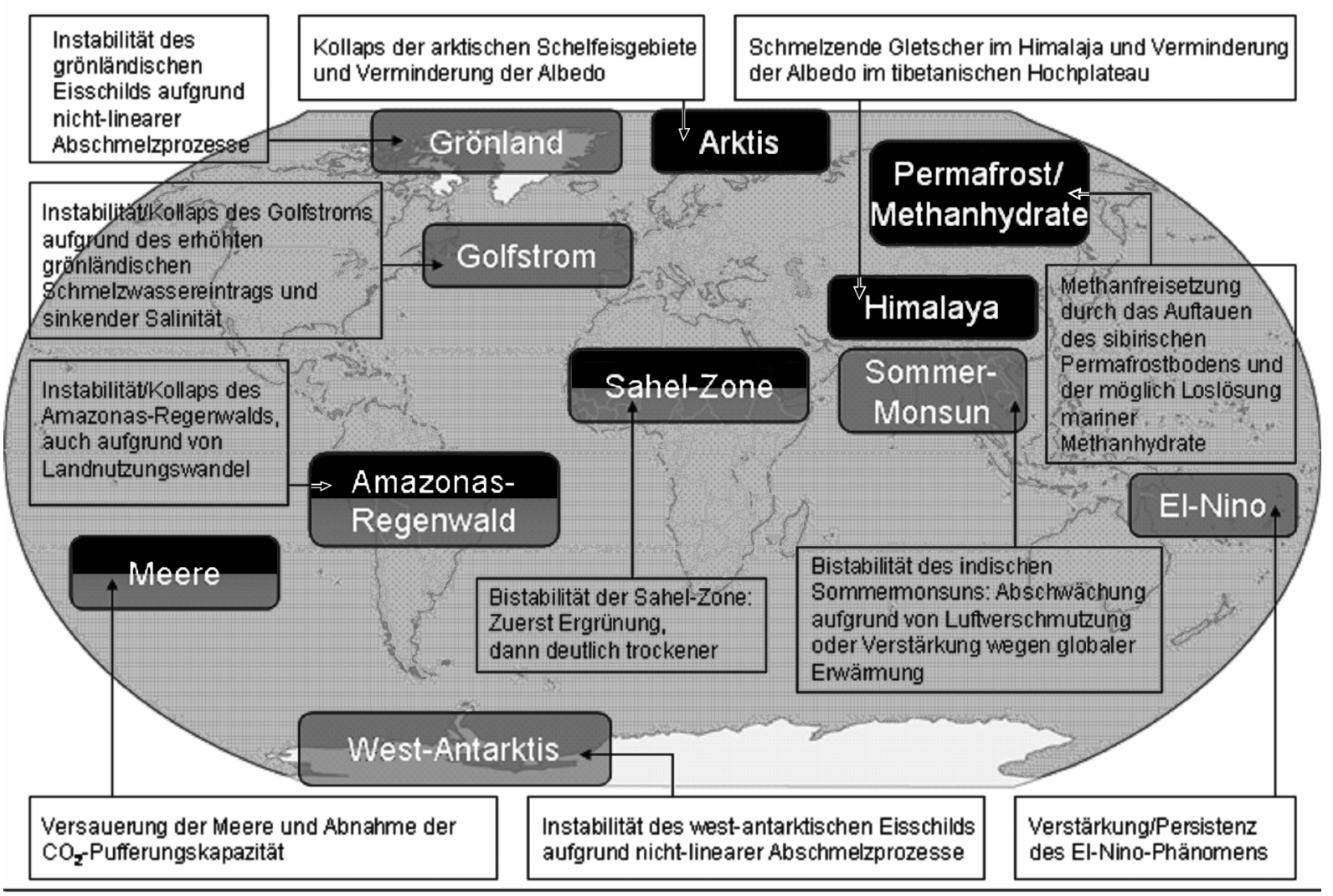

Klima-Kipp-Elemente mit direkten und großen Folgen für Menschen

Klima-Kipp-Elemente mit positiver Rückkopplung auf Temperatur

Quelle: Germanwatch auf Grundlage von Schellnhuber, 2005

haben ihren Ursprung in ein und dem selben Eisfeld. Und 40 Prozent der Erdbevölkerung bekommt 50 Prozent oder mehr ihres Wassers von diesen schmelzenden Eisformationen.“

Vor diesem Hintergrund wird zum einen deutlich, warum in der Wissenschaft ein heftiger Disput ausgebrochen ist, ob das an sich schon ehrgeizige Ziel der Europäischen Union (EU), den globalen Temperaturanstieg auf weniger als zwei Grad gegenüber vorindustrieller Zeit ${ }^{8}$ zur Gefahrenabwehr zu begrenzen, ausreichend sei. ${ }^{9}$ Hierauf bezog sich der Vorsitzende des IPCC, R.K. Pachauri, in seiner Eröffnungsrede des UN-Klimagipfels in Posen (COP 14) im Dezember 2008 , als er erklärte, der Temperaturanstieg müsse möglicherweise auf 1,5 Grad C beschränkt werden, um einen gefährlichen Klimawandel zu vermeiden. Andere Wissenschaftler fordern inzwischen, den Temperaturanstieg auf ein Grad zu begrenzen. ${ }^{10}$ Zum anderen erklärt sich, warum viele Wissenschaftler und Politiker alarmiert sind: Weil der Emissionsanstieg seit dem Jahr 2000 auf einem Niveau liegt, das zu einem Temperaturanstieg von mehr als fünf bis sechs Grad in diesem Jahrhundert führen könnte.

8 Gegenüber dem Jahr 2000 bedeutet das einen Temperaturanstieg von etwa 1,3 Grad C.

9 Hansen J., Sato M., Kharecha P., Lea D. W., Siddall M.: Climate Change and Trace Gases, in: Philosophical Transactions of the Royal Society, 2007, 365, S. 1925-1954.

10 W. L. Hare: A Safe Landing for the Climate, 2009; in: The Worldwatch Institute, State of the World 2009, Into a Warming World, Washington D.C.

\section{Zusammenhänge zwischen Klima-, Energie- und Ernährungssicherheit}

Der Zusammenhang von drei Krisen, die die Welt zunehmend in Atem halten, wird immer offenkundiger: die Klimakrise, die Energiekrise und die Ernährungskrise.

Die Nutzung fossiler Energieträger ist seit der industriellen Revolution Grundlage des Wohlstands der Industrie- und zunehmend auch der Schwellenländer. Doch immer mehr Argumente sprechen für einen Wechsel zu einem emissionsarmen Wohlstandsmodell jenseits der fossilen Energieträger. Der neue US-Präsident Barack Obama hat bereits in seiner Antrittsrede die Herausforderung verdeutlicht, die in der Abhängigkeit von fossilen Energieträgern liegt. „Jeder Tag bringt weitere Beweise, dass die Art, wie wir Energie verbrauchen, unsere Gegner stärkt und unseren Planeten bedroht." Beim ersten Besuch von USAußenministerin Clinton in China standen Klima- und Energiesicherheit ganz oben auf der Agenda. ${ }^{11}$

Wenige Wochen zuvor, im November 2008, hatte Nobuo Tanaka, Geschäftsführer der Internationalen Energieagentur (IEA)

11 Vgl. auch die vom neuen US Energieminister Steven Chu vor seiner Ernennung mit erarbeitete Roadmap: Chu S., Thornton J.: A Roadmap for U.S.-China Cooperation on Energy and Climate Change, Hg. Pew Center, Asia Soceity, January 2009. 
bei der Vorstellung des neuen World Energy Outlook (WEO) gewarnt: „Die gegenwärtigen Trends in der Energieversorgung und im Energieverbrauch sind grundlegend nicht nachhaltig - weder ökologisch, noch ökonomisch noch sozial. Sie können und müssen geändert werden. Wachsende Importe von Öl und Gas in die OECD-Staaten und ins sich entwickelnde Asien zusammen mit der Konzentration der Produktion in einer kleinen Zahl von Ländern würde das Risiko von Angebotsunterbrechungen und starken Preissprüngen vergrößern. Gleichzeitig würden die Treibhausgasemissionen exorbitant steigen. Diese würden die Welt auf einen Temperaturanstieg von sechs Grad Celsius zusteuern lassen."12. Der Gaspreiskonflikt zwischen Russland und der Ukraine mit erheblichen Konsequenzen auch für andere Lieferländer hat einen weiteren Vorgeschmack auf die sich zuspitzende Energiekrise gegeben. Die Internationale Energieagentur hat gewarnt, dass es bei einer sich erholenden Wirtschaft schnell zu Preissprüngen beim Ölpreis kommen könnte. „Eines ist sicher“, so Tomaka, „während Marktungleichgewichte die Volatilität steigern, ist die Ära des billigen Öls vorbei."

Die beiden Themen Klima- und Energiesicherheit werden sinnvollerweise gemeinsam diskutiert. Auf der einen Seite gibt es vielfältige Synergien bei der Lösung der beiden Probleme, auf der anderen Seite gibt es Spannungen, die überbrückt werden müssen. Energiesparen, Energieeffizienz und erneuerbare Energien dienen sowohl der Energie- als auch der Klimasicherheit. ${ }^{13}$ Aus sicherheitspolitischer Sicht gebührt Strategien, die diese Lösungsansätze voranbringen, der Vorrang. Teil einer solchen Strategie ist auch der Ausbau des Stromnetzes. Zum einen könnten wesentlich größere Mengen dezentral erzeugten Stroms aus erneuerbaren Energien aufgenommen und ein intelligentes Laststrommanagement sowie Laststeuerung ermöglicht werden (Smart Grid). Zum anderen könnte durch Hochgeschwindigkeits-Übertragungsleitungen (HGÜ) mit wenig Wirkungsverlust Strom aus Regionen mit kostengünstigen Potenzialen importiert werden (Super Grid) ${ }^{14}$.

Bei den meisten Energieträgern sind die Probleme offensichtlich. Kohle wird in verschiedenen Regionen der Welt für die nächsten Jahrzehnte als unverzichtbar für die Energiesicherheit betrachtet. Zugleich ist sie der Energieträger mit dem größten $\mathrm{CO}_{2}$-Ausstoß pro Energieeinheit. Aus Gründen der Klimasicherheit liegt es deshalb nahe, auf die Verbrennung von Kohle zu verzichten oder aber sie nur noch in solchen Kohlekraftwerken zu verbrennen, in denen das $\mathrm{CO}_{2}$ abgetrennt und geologisch tief gelagert, anstatt in die Atmosphäre abgegeben wird. In kaum einem Szenario, das ehrgeizige $\mathrm{CO}_{2}$-Ziele erreichen will, kann auf diese CCS-Technologie (Carbon Capture and Storage) verzichtet werden. Der großtechnische Test, ob diese Technologie hält, was sie verspricht, liegt allerdings noch vor uns.

12 New Energy Realities - WEO Calls for Global Energy Revolution Despite Economic Crisis, 12 November 2008, London; http://www.iea.org/textbase/ press/pressdetail.asp?PRESS_REL_ID=275, eigene Übersetzung.

$13 \mathrm{Im}$ Jahr 2006 hat die damalige britische Außenministerin Margret Beckett den Begriff "Klimasicherheit" in die internationale Debatte eingeführt. Beckett, M.: Foreign Policy and Climate Security, Berlin Speech, 24.10.06, http://www. fco.gov.uk/servlet/Front?pagename=OpenMarket/Xcelerate/ShowPage\&c=Pa ge\&cid=1007029391647\&a=KArticle\&aid $=1161588023142$.

14 Vgl. für die europäische Debatte: Battaglini A., Lilliestam J., Bals C. and Haas, A: The SuperSmart Grid, June 18th 2008; Hg. European Climate Forum, Potsdam Institute for Climate Impact Research.
Gas wird wegen des relativ geringen $\mathrm{CO}_{2}$-Ausstoßes pro Energieeinheit aus Klimasicht als Brückentechnologie betrachtet, vor allem, wenn es in hoch effizienten Kraft-Wärme-Kopplungsanlagen zum Einsatz kommt. Allerdings hat die jüngste RusslandUkraine-Krise auch die Verletzlichkeit in der Versorgung mit diesem Energieträger vor Augen geführt. Die Abhängigkeit von russischen Gaslieferungen wird in Europa allerdings bisweilen überbetont. Russland ist mindestens im gleichen Ausmaß von den Energieeinnahmen aus der EU abhängig, wie diese vom russischen Gas.

Die Kernkraft wird von verschiedenen Akteuren als eine Lösung für das Klimaproblem ins Spiel gebracht. Sie vernachlässigen dabei die ungelösten Fragen der Endlagerung radioaktiven Abfalls sowie deren ungeklärte Kosten. Aber aus Klimasicht kann der Beitrag der Atomenergie international nur gering sein. Noch kritischer fällt die Bewertung aus sicherheitspolitischer Sicht aus. Es reicht, einen Blick auf die spannungsreichen Konstellationen mit dem Iran, mit Nordkorea und mit Pakistan zu werfen, um zu sehen, welche Sicherheitsrisiken sich aus der vermeintlich nur für die Stromerzeugung genutzten Kernenergie ergeben. Neben der Herstellung von Nuklearwaffen wird zunehmend auch die Gefahr des Nuklear-Terrorismus diskutiert.

In jüngster Zeit zeigt sich vermehrt, dass auch die Ernährungsund Wassersicherheit immer enger sowohl mit der Klima- ${ }^{15}$ als auch mit der Energiesicherheit verknüpft sind. Der Klimawandel kann sich einerseits durch schleichende Veränderungen wie den Meeresspiegelanstieg und die Wüstenbildung, andererseits durch plötzliche Abweichungen wie Extremwettersituationen und drittens durch zukünftig mögliche Kipp-Punkte negativ auf die Wasser- und Ernährungssicherheit auswirken. Es wäre somit sinnvoll, die Debatten und Strategien zur Begrenzung der Konsequenzen des Klimawandels auf ihre Relevanz für das Recht auf angemessene Ernährung auf globaler, nationaler sowie Haushaltsebene abzuklopfen ${ }^{16}$ und die Anpassungsstrategie dementsprechend auszurichten.

Bio- bzw. Agrofuels werden in erster Linie aus Gründen der Energiesicherheit, nicht aus Gründen der Klimapolitik propagiert. Die Energiedichte ist zu gering, um klimapolitisch eine wichtige Rolle spielen zu können. Zum Teil, etwa wenn Regenwälder hierfür gerodet werden, kann die Klimabilanz sogar deutlich negativ sein. Gravierender aber können die Konsequenzen für die Ernährungssicherung sein. Allerdings ist die Auswirkung ambivalent, denn während Produzenten - die Bauern und Verarbeiter - von höheren Preisen profitieren, wirken sich diese für Konsumenten negativ aus. Die Preissprünge, die als einer von mehreren Faktoren auch vom Biosprit-Boom ausgelöst worden sind, haben die Zahl der Hungernden wieder ansteigen lassen. Die gegenwärtige Wirtschaftskrise bewirkt einen weiteren kräftigen Anstieg der Zahl der Hungernden. Derzeit hungern mehr als eine Milliarde Menschen.

\footnotetext{
15 Für eine detaillierte Diskussion des Zusammenhangs von Globalem Klimawandel und dem Recht auf Nahrung siehe Windfuhr M., Bals C., Harmeling S.: Climate Change Food Security and The Right to Adequate Food, Hg. Diakonie Katastrophenhilfe, Germanwatch, Brot für die Welt, Stuggart, Bonn, 2008.
} 
Abbildung 2: Das für die humanitäre Sicherheit zentrale Zusammenspiel und die Gegenpole zwischen den klimarelevanten Aspekten Klima-, Energie- und Ernährungssicherheit

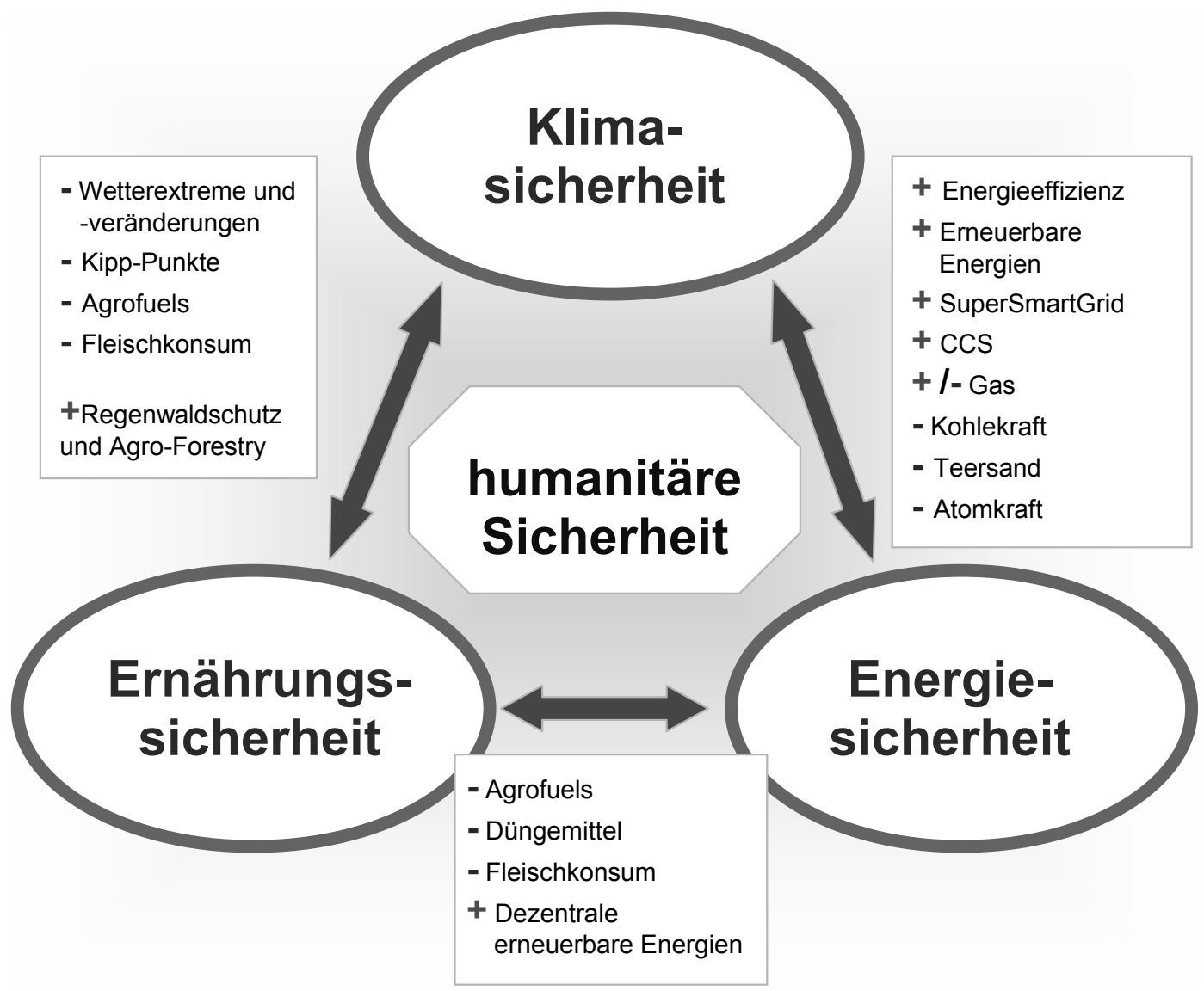

Quelle: Germanwatch

Gravierender könnte sich allerdings in der Zukunft auswirken, dass durch den Biosprit Getreide prinzipiell zum Ersatzstoff für Erdöl geworden ist. Es ist daher damit zu rechnen, dass die Getreidepreise ab einem gewissen Anstieg des Ölpreises alle Preissprünge des schwarzen Goldes mitmachen werden. Diese mögliche extreme Volatilität des Getreidepreises würde die Ernährungssicherheit auf der Erde drastisch verschlechtern.

Ein weiteres Problem ergibt sich aus dem weltweit schnell wachsenden Fleischkonsum. Die Fleischproduktion ist um ein Vielfaches energie- und flächenintensiver als der Anbau von Getreide und Gemüse. Die massive Umstellung auf Fleischernährung, insbesondere in China und Indien, führt wegen des hohen Flächen- und Futtermittelbedarfs zu steigenden Nahrungsmittelpreisen und zur Abholzung der Regenwälder. Allerdings ist der Fleischkonsum pro Kopf dort wesentlich geringer als etwa in Deutschland. Auch für die hiesige Fleischwirtschaft, insbesondere seit dem BSE-Skandal, wird viel Soja aus Entwicklungsländern als Futtermittel importiert.

Synergien zwischen Klima- und Ernährungssicherheit gibt es hingegen bei Strategien zum Schutz des Regenwaldes. Der Wald nimmt im Klimasystem den Stellenwert einer der wichtigsten $\mathrm{CO}_{2}$-Senker ein, stützt gleichzeitig den Wasserkreislauf, verringert die Erosion, erhält die Biodiversität und den Lebensraum für die dortige Bevölkerung. Agroforestry kann einen Beitrag zur Ernährungssicherung und für den Erhalt des Regenwaldes spielen.

Die gegenwärtige Finanzkrise hat dafür sensibilisiert, nicht nur die Risiken erster Ordnung, sondern auch die möglicherweise noch wesentlich dramatischeren Risiken zweiter oder dritter Ordnung zu beachten. ${ }^{17}$ Die Krise der Immobilienfinanzierung konnte noch als regional begrenzte Krise erscheinen. Viele Regierungen, deren Banken nicht direkt betroffen waren, vertraten die Ansicht, die Krise habe für sie nur geringe Bedeutung. Doch die diversen Rückkopplungen bewirkten nicht nur eine Krise des Finanzsektors, sondern der gesamten Wirtschaft und hatten damit auch Folgen für die Arbeitsplätze in immer mehr Staaten. Ebenso besteht angesichts eskalierender Klima-, Energie- und Ernährungskrisen die Gefahr, dass die Risiken zweiter oder dritter Ordnung weit gravierender als die bislang diskutierten Konsequenzen erster Ordnung sind. Diese könnten etwa umfangreiche Fluchtbewegungen, erneute Finanzmarktkrisen, Öko-Diktaturen oder Maßnahmen zur Wetter- bzw. Klimabeeinflussung (Geo-Engineering), die gewollte oder nicht-gewollte Nebenwirkungen auf andere Staaten haben könnten, auslösen bzw. fördern.

17 Für Diskussionen zu diesem Punkt danken wir vor allem Armin Haas (PIK) und Matthias Onischka (Wuppertal Institut). 
Neben der Nutzung von Synergien liegt eine große Chance darin, die drei Sicherheitsrisiken Klima, Energie und Ernährung als Einheit zu behandeln. Insbesondere das Klimaproblem, aber global gesehen auch die Ernährungsfrage, lässt sich nur durch Kooperation zwischen Staaten, nicht aber mit militärischen Mitteln lösen. Ein doppelter Fokus ist zentral: Das Unbewältigbare vermeiden - also eine aktive Klimaschutzpolitik. Das Unvermeidbare bewältigen - also eine aktive Politik der armutsorientierten Anpassung, Resilience und Bildung. Fatal wäre hingegen, wenn Energiesicherheitspolitik stattdessen auf eine Militarisierung der Klimapolitik setzen würde. Zentral sollte vielmehr eine Strategie sein, die günstige Rahmenbedingungen für friedliche Lösungen in den Mittelpunkt stellt. ${ }^{18}$

„Das Schlüsselwort unseres Jahrhunderts heißt Zusammenarbeit. " ${ }^{19}$ Es ist ermutigend zu sehen, dass der neue US-Präsident Barack Obama ganz ähnlich argumentiert: , Es gibt zur Partnerschaft und Zusammenarbeit zwischen den Nationen keine Alternative. Es ist der Weg, der einzige Weg, um unsere gemeinsame Sicherheit zu gewährleisten. "20 Dies ist nach einer schwierigen Phase der transatlantischen Beziehungen eine Chance für eine Neuorientierung.

Es gibt also Anzeichen dafür, dass die EU und die USA in ihrer Klima- und Energiesicherheitspolitik die Chancen der Kooperation zukünftig in den Vordergrund stellen könnten. Auch die Tatsache, dass insbesondere Herausforderungen für die Wasserversorgung eher zu stabilisierenden Kooperationen zwischen Staaten als zu Konflikten geführt haben, zeigt das Potenzial von kooperativen Ansätzen. ${ }^{21}$ So haben etwa Konflikte um Wasser in keinem der 263 grenzüberschreitenden Flussgebiete zu einem internationalen Krieg geführt. ${ }^{22}$

Für Deutschland und die EU bieten sich Chancen, durch eine Vorreiterrolle in der globalen Klimapolitik, in der Entwicklungszusammenarbeit und in der Energiepolitik, weltpolitische und wirtschaftliche Gestaltungsmacht zu gewinnen. ${ }^{23}$ In der sogenannten Solana-Strategie der EU, dem ersten Versuch, sich der Herausforderung der Klimasicherheit systematisch zu stellen, heißt es: „, Die EU ist aufgrund ihrer führenden Rolle in der Entwicklungspolitik und der globalen Klimapolitik sowie aufgrund des breiten Spektrums der ihr zur Verfügung stehenden Instrumente in einzigartiger Weise dafür gerüstet, auf die Auswirkungen des Klimawandels auf die internationale Sicherheit zu reagieren. Bei der Sicherheitsherausforderung kommen zudem die Stärken Europas zur Geltung, denn Europa verfügt über ein umfassendes Konzept zur Konfliktverhütung, Krisenbewältigung und Konfliktnachsorge und gehört zu den

18 Wir danken Anja Köhne, bis vor kurzem Germanwatch-Vorstandsmitglied, für die Diskussion.

19 Schmidt H., von Weizsäcker R, Bahr E. und Genscher H.-D., Für eine atomwaffenfreie Welt, FAZ, 8. Januar 2009.

20 Obama, Barack: "A World That Stands as One", Speech in Berlin Germany, 24 July, 2008, unter: http://my.barackobama.com/page/content/berlinvideo/.

21 Vgl. auch: Taenzler, D.: Entwicklungsrisiko Klimawandel: Die Notwendigkeit kooperativer Ansätze, Diskussionspapier: Stiftung Wissenschaft und Politik, Januar 2009.

22 Vgl. Wolf, A: A Long Term View of Water and Security: International Waters, Natioal Issues and Regional Tensions, Berlin 2007.

23 Vgl. Bauer S., Messner D., 2007: Der Klimawandel bedroht globale Entwicklung und internationale Stabilität, DIE-Analysen und Stellungnahmen 7/2007, Bonn.
Hauptbefürwortern eines wirksamen Multilateralismus. “24 Sind die EU und Deutschland aber diesen Herausforderungen gewachsen?

\section{Klima- und Energiesicherheitspolitik in Europa}

Im März 2007 läutete die EU eine neue Phase der Klimasicherheitspolitik ein. Am 14. März legten Solana und die EU-Kommission das Strategiepapier „Climate Change and International Security“ vor. Der Klimawandel wird hier in erster Linie als „Bedrohungsmultiplikator“ (threat multiplier) thematisiert, der bestehende Bedrohungen verschärft, vor allem in fragilen und bereits von Konflikten betroffenen Regionen. Nach der Diskussion der Bedrohungen und Schwerpunktregionen wird der Schluss gezogen, dass der Klimawandel als Thema der Außenpolitik „sogar zu einer positiven Triebfeder für die Verbesserung und Umgestaltung der globalen Struktur- und Ordnungspolitik werden“ kann.“ ${ }^{25}$ Für die EU bedeute dies, „ihre Führungsrolle aufrechterhalten und verstärkt darauf hinwirken, dass im Jahr 2009 ein ehrgeiziges Übereinkommen für die Zeit nach 2012 geschlossen wird“. An diesem Anspruch gilt es die EU-Klimapolitik bis Ende 2009 zu messen.

Am 9. März 2007 legte die EU schließlich den Grundstein für eine sehr ambitionierte Klima- und Energiepolitik. Ergebnis des Frühlingsgipfels der Staats- und Regierungschefs unter dem Ratsvorsitz von Angela Merkel war ein anspruchvolles Energieund Klimapaket: Unabhängig von dem Engagement anderer Staaten sollen die Treibhausgase in Deutschland bis 2020 gegenüber 1990 um mindestens 20 Prozent verringert werden. Wenn die anderen Industrie- und Schwellenländer mitziehen, dann sollen es sogar 30 Prozent werden - als verbindliches Ziel. Darüber hinaus ist beabsichtigt, den Anteil erneuerbarer Energien am Energieverbrauch von heute sechs auf 20 Prozent steigern sowie die Energieeffizienz um 20 Prozent gegenüber einem „Weiter so“ zu verbessern. „Die Brüsseler Entscheidung geht in ihrer Bedeutung weit über den Klimaschutz hinaus. Europa, das bereits durch eine multilaterale Kooperationspolitik den Kalten Krieg friedlich überwunden hat, stellt sich seiner globalen Verantwortung und dokumentiert in einer Überlebensfrage der Menschheit Handlungsfähigkeit“26, bescheinigte der frühere Außenminister Hans-Dietrich Genscher nach diesem Frühlingsgipfel.

Als die Regierungschefs der EU-Mitgliedstaaten Ende 2008 die zur Umsetzung dieser Ziele notwendigen Gesetzespakete verabschieden sollten, wankte die EU angesichts der Finanz- und Wirtschaftskrise, doch sie fiel nicht. Die Ziele blieben erhalten. Allerdings gab es für den Klimaschutz bzw. die wirkungsvolle Umsetzung schmerzhafte Kompromisse: Zum einen darf für

24 Klimawandel und internationale Sicherheit. Papier des Hohen Vertreters und der Europäischen Kommission für den Europäischen Rat, S113/08, Brüssel 4 März 2008, unter: http://www.consilium.europa.eu/uedocs/cms_data/docs/ pressdata/DE/reports/99391.pdf.

25 Council of the European Union, REPORT from the Commission and the Secretary-General/High Representative to European Council: Climate Change and international security, Brussels, 3.3.2008;

26 Bals C., Hamm H., Jerger I., Milke K.: Die Welt am Scheideweg, Reinbek 2008, S. 166 
den Zeitraum 2008 bis 2020 ein allzu großer Anteil der Vorgaben durch Zertifikate aus Entwicklungsländern eingehalten werden. Zum anderen gab es für die Industrie in ganz Europa und die Energiewirtschaft in Osteuropa großzügige Ausnahmen von der Vorgabe Emissionserlaubnisse vollständig zu versteigern.

Auch für die $\mathrm{CO}_{2}$-Abscheidung und geologische Tiefenlagerung (CCS) wurde eine Richtlinie verabschiedet. In den Nationalstaaten, auch in Deutschland, wird diese gegenwärtig in nationales Recht umgesetzt. 12 große Pilotanlagen sollen vor 2020 in der EU gebaut werden. Zugleich bereitet die EU derzeit die Rahmensetzung für CCS-Kooperationen mit Schwellenländern vor, denn dort wird der eigentliche Nutzen dieser Technologie erwartet, wenn sie denn hält, was sie verspricht.

Am 8. Januar 2009 hat die EU-Kommission ein neues GreenPaper $^{27}$ vorgestellt, um bei der zügigen Behebung eines großen Hemmnisses auf dem Weg zu einer weit ambitionierteren Versorgung der EU mit erneuerbaren Energien voranzukommen: „Die europäischen Energienetze sind die Lebensadern, die die Energie für unsere Wohnungen, Unternehmen und Freizeitaktivitäten liefern.." So könnte einerseits ein regional weit gespanntes Netz zwischen Nordeuropa (Off-Shore-Wind), Mittel- und Osteuropa (Biomasse), Nordafrika (Solarthermische Kraftwerke und Wind) sowie der EU entstehen. Genauso aber bedarf es des intelligenten Ausbaus des Stromnetzes, um in großem Maße dezentralen erneuerbaren Strom ins Netz einzuspeisen. Nur so können Schwankungen ausgeglichen werden, die für solche Einspeisungen typisch sind.

Bei ihrem Frühlingsgipfel im März 2009 haben die EU-Regierungschefs versäumt, international ein eindeutiges Signal auszusenden, dass die EU im Jahr des angepeilten Post-2012/Kopenhagen-Abkommens für den internationalen Klimaschutz weiter die Führungsrolle übernehmen wird. Als Grundarchitektur für dieses Abkommen war beim Start der Verhandlungen beim UN-Klimagipfel in Bali im Dezember 2007 Folgendes vereinbart worden ${ }^{28}$ : Die Industriestaaten legen ambitionierte Pläne vor, wie sie den Klimaschutz national umzusetzen beabsichtigen. Zugleich präsentieren die Entwicklungs- und Schwellenländer umfassende finanzielle und technische Kooperationsmodelle. Als Prinzip gilt: Je ambitionierter die Klimaschutzpläne, desto umfassender die Finanz- und Technologiekooperation. Zahlreiche Schwellenländer haben inzwischen solche Klimaschutzpläne erarbeitet. Doch die Industrieländer mauern. Sie sind nicht bereit anzugeben, wie viel Geld sie investieren wollen und wie die notwendigen Finanzmittel aufgebracht werden sollen. Der UN-Klimagipfel Ende 2008 endete im Eklat, weil es in der Finanzierungsfrage von Seiten der Industrieländer keine Bewegung gab. ${ }^{29}$ Insgesamt wird der Finanzbedarf für Klimaschutz, Waldschutz und Anpassung an den Klimawandel auf

27 Kommission der Europäischen Gemeinschaften, Grünbuch hin zu einem sicheren, nachhaltigen und wettbewerbsfähigen europäischen Energienetz Brüssel, den 13.11.2008, KOM(2008) 782 endgültig, \{SEC(2008)2869\}

28 UNFCCC Bali Action Plan, Decision -/CP.13, December 2007, insbes. 1 b ii; Siehe zu den Hintergründen dieser hart umkämpften Entscheidung: - Bali 2007 - dramatisches Ringen um die Zukunft - Eine Reportage, in Bals C., Hamm H., Jerger I., Milke K., 2008, S. 19-33.

29 Bals, C.: Klimazug im ,Tal des Todes‘ zwischen Posen und Kopenhagen, Bonn 2009. etwa 110 Milliarden US-Dollar jährlich geschätzt. Auf die EU kämen etwa 35 Milliarden US-Dollar jährlich zu.

Am 20. März 2009 konnten sich die EU-Regierungschefs aber nicht dazu durchringen, das notwendige Signal hierfür zu geben. Zahlen, über die man zu verhandeln bereit wäre, wurden nicht genannt. Es wurde lediglich festgehalten, dass man bereit sei, „den fairen Anteil“ der EU für diese Finanzierung zu leisten. Für einen Vorreiter, der die Messlatte für die anderen Industrieländer auflegen soll, ist das zu wenig. Immerhin hat man sich in den Schlussverhandlungen durchgerungen, über Instrumente zu diskutieren, mit deren Hilfe die notwendige Finanzierung bewerkstelligt werden könnte. Hierzu zählen erstens ein Ansatz, der die Pflichten nach Kriterien wie Emissionsausstoß und Wirtschaftskraft aufteilen würde, zweitens die Versteigerung von Emissionserlaubnissen durch Staaten oder im Rahmen von Emissionshandelssystemen, drittens eine Kombination aus beiden. Im Entwurf des Textes wird jedoch eine Bereitschaft über solche Instrumente zu verhandeln nicht deutlich. Die EU-Regierungschefs kündigen lediglich an, dass sie „rechtzeitig vor Kopenhagen“ ihre konkreten Angebote auf den Tisch legen werden. Doch „rechtzeitig“ wäre zu jenem Zeitpunkt gewesen. Denn von März bis Juni soll insgesamt vier Wochen lang verhandelt werden, ohne entsprechende Angebote werden diese Verhandlungen kaum von der Stelle kommen.

Vielfach war befürchtet worden, dass die Wirtschaftskrise die Klimaschutzambitionen für das angepeilte Kopenhagen-Abkommen Ende 2009 deutlich herunterschrauben würde. Tatsächlich liegt das Schwächeln der EU in der Debatte über diese Frage begründet. So hatte etwa Silvio Berlusconi bei den Beratungen im Dezember 2008 über das Energie- und Klimapaket geklagt, es sei „absurd, in Zeiten der Krise über den Treibhausgas-Ausstoß zu reden - das ist, als ob jemand, der Lungenentzündung hat, über eine Dauerwelle nachdenken würde. “ Andere halten dagegen, es sei absurd auf eine Krise des „Pump-Kapitalismus" (Dahrendorf) mit der Anhäufung von ökologischen Schulden zu reagieren; auf ein Versagen des Risikomanagements mit dem Verzicht auf Risikomanagement zu antworten. „Wir müssen zwei Krisen mit einer Klatsche schlagen“, meint Klaus Töpfer. Auch der EU-Kommissionspräsident Jose Manuel Barroso wird nicht müde zu betonen, dass die Wirtschaftskrise keine Entschuldigung darstelle, den Klimawandel nicht weiter zu bekämpfen. ${ }^{30}$ Für viele überraschend hat der neue US-Präsident Obama am 12.02.2009 in seiner ersten Rede vor beiden Häusern angekündigt, die Energie- und Klimasicherheit gemeinsam mit der Gesundheits- und Bildungspolitik ins Zentrum seiner Pläne zur Bekämpfung der Wirtschaftskrise zu stellen. Er kündigte eine Verdoppelung des Einsatzes erneuerbarer Energien in den USA in nur drei Jahren an. „Aber um wirklich unsere Wirtschaft zu transformieren, um unsere Sicherheit zu schützen und um unseren Planeten vor den Konsequenzen des Klimawandels zu bewahren, müssen wir letztlich saubere, erneuerbare Energien zur profitabelsten Energieform in Amerika machen. Deshalb fordere ich den Kongress auf, mir eine

\footnotetext{
30 "If we do not take effective actions to tackle the climate change, there will be larger threats. The financial crisis must not become an excuse for not properly dealing with the climate change." Pressekonferenz des siebten Asia-Europe Meetings (ASEM), 25. Oktober 2008.
} 
Gesetzgebung mit einer marktbasierten Emissionsobergrenze und entsprechenden Anreizen für Erneuerbare Energien zu senden. "Seit allerdings das Haushaltsbüro des Kongresses errechnet hat, ${ }^{31}$ dass mit den Schulden für das Konjunkturprogramm und für Obamas ersten Haushalt mit Ausgaben von insgesamt nicht weniger als 3,6 Billionen US-Dollar das Etat-Defizit in den kommenden zehn Jahren wohl niemals unter 4,1 Prozent des Bruttoinlandprodukts sinken dürfte, gerät das Ausgabenprogramm für Klimaschutz, Bildung und Gesundheit zunehmend unter Druck. Ob sich die Klimaschutz-Ansätze in den Stimulus-Paketen der Industrie- und Schwellenländer durchsetzen, wird maßgeblich darüber entscheiden, wie ambitioniert ein Kopenhagen-Abkommen ausfallen kann. Es gilt, die Investitionen in die Infrastruktur für und nicht gegen den Klimaschutz zu investieren. Denn allzu naiv wäre die Vorstellung, der Zug der fortschreitenden Zerstörung der Überlebensbedingungen würde Geschwindigkeit und Richtung verändern, indem wir lediglich in seinem Inneren gegen die Fahrtrichtung laufen.

Innerhalb der EU wiederum wird bis Ende 2009 besonders viel von den Regierungen Dänemarks, Schwedens und Deutschlands erwartet. Die dänische Regierung ist Gastgeber des wichtigen Gipfels von Kopenhagen. Schweden wird in der zweiten Jahreshälfte die EU-Präsidentschaft innehaben. Es ist zu hoffen, dass sie diese Rolle engagierter im Sinne eines ambitionierten Klimaschutzes ausübt als die jetzige tschechische Ratspräsidentschaft. Da die Verhandlungsposition, was die EU-Reduktionsziele angeht, festgeklopft ist, wird es vor allem darauf ankommen, die Bereitschaft zu einer groß angelegten Finanz- und Technologiekooperation zu stärken. In Schweden selbst ist das

31 Vgl. Klüver, R.:Große Versprechen, Kleines Geld, in: SZ, 23.3.09, S. 4.
Finanzministerium einstweilen noch ein Bremsklotz auf dem Weg dorthin.

Entscheidend für die Entwicklung der EU-Position ist die Rolle Deutschlands. „Ohne Deutschland lässt sich die notwendige ambitionierte Positionierung in der EU nicht organisieren“, erklärte jüngst die dänische Umweltministerin. Das Finanzministerium bremst hier bei allem, wo öffentliche Gelder bereitgestellt werden sollen, das Wirtschaftsministerium, wenn es darum geht, dass die Wirtschaft nach dem Verursacherprinzip zur Kasse gebeten werden soll. Angesichts des weit höheren Pro-Kopf-Emissionsausstoßes, den hohen Emissionen in der Vergangenheit und des größeren finanziellen Spielraums - trotz Finanzkrise - stehen die Industrieländer in der Pflicht zu zeigen, dass sie das Wort „Kooperation“ mit Leben füllen. Um die Widerstände insbesondere Polens, Ungarns, Bulgariens und Italiens zu überwinden, bedarf es einer Führungsrolle Deutschlands in der EU, und das mitten im nationalen Wahlkampf.

Die G8-, G20-Gipfel und die Treffen der großen Emittenten (MEM) in diesem Jahr sind Glaubwürdigkeitstests für eine ernst gemeinte Strategie der Klima- und Energiesicherheit der EU und Deutschlands. Neben dem US-Präsidenten ist hier die deutsche Kanzlerin mit dem höchsten Erwartungsdruck konfrontiert. Zu den günstigen Rahmenbedingungen für ein ambitioniertes Abkommen in Kopenhagen gehören auch intensive bilaterale Absprachen der EU und Deutschlands insbesondere mit den USA, China, Indien, Russland und den Staaten Afrikas. Wer jetzt angesichts der Finanz- und Wirtschaftskrise eine zielstrebige Strategie der Klima- und Energiesicherheit zur Schönwetterpolitik erklärt, der legt den Grundstein für die Klimaprobleme der Zukunft.

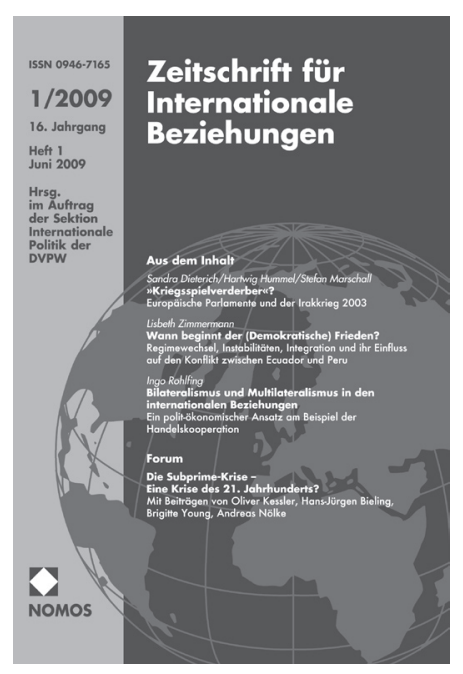

\author{
Zeitschrift für Internationale Beziehungen (ZIB) \\ 16. Jahrgang 2009, 2 Ausgaben pro Jahr. \\ ISSN 0946-7165 \\ Jahresabo: 72,- $€^{*}$ \\ Jahresabo für Mitglieder der DVPW: 64,- $€^{*}$ (bitte Bescheinigung zusenden) \\ Jahresabo für Studierende: 40,- $€^{*}$ (bitte Bescheinigung zusenden) \\ ${ }^{*}$ Preis zzgl. Vertriebs-/Direktbeorderungsgebühren Inland $(6,42 € / 1,61 €) 8,03 €$ \\ inkl. MwSt., jährlich. Kündigung 3 Monate vor Kalenderjahresende möglich.
}

Die „ZIB“ bildet das führende deutschsprachige Kommunikationsforum für die politikwissenschaftliche Analyse internationaler Politik. Mit ihrem spezifischen Profil in der Verknüpfung von Theorie und Empirie sowie durch herausragende Beiträge genießt sie weithin Beachtung. 